\title{
Experimentelle Erkundung von Wildbächen, Murgängen, Hangrutschungen und Steinschlag: Aktuelle Beispiele der WSL
}

\author{
M. Stähli ${ }^{1}$, C. Graf ${ }^{1}$, C. Scheidl ${ }^{2}$, C. R. Wyss ${ }^{1}$, and A. Volkwein ${ }^{1}$ \\ ${ }^{1}$ Eidg. Forschungsanstalt für Wald, Schnee und Landschaft WSL, Birmensdorf, Schweiz \\ ${ }^{2}$ Institut für Alpine Naturgefahren, Universität für Bodenkultur, Wien, Österreich
}

Correspondence to: M. Stähli (manfred.staehli@wsl.ch)

Received: 12 May 2014 - Revised: - - Accepted: 24 July 2014 - Published: 26 January 2015

\begin{abstract}
Kurzfassung. Experimente werden oft als selbstverständlicher Bestandteil der Erforschung von Massenbewegungen betrachtet. Häufig sind wir uns jedoch nicht bewusst, was genau die Rolle der Experimente in der ganzen Wissensbildung und der Anwendung für die Praxis ist. Der vorliegende Artikel soll dies anhand von aktuellen Beispielen der Eidg. Forschungsanstalt WSL aufzeigen. Ein Laborexperiment zur Frage der Kurvenüberhöhung von Murgängen erlaubt einen detaillierten Einblick in einen Vorgang, den man natürlicherweise im Feld nie gewinnen könnte. Ein Laborrinnenexperiment ermöglicht die Generalisierung von Methoden und Beobachtungen zum Sedimenttransport in Fliessgewässern. Ein Steinschlag-Experiment stellt eine essentielle Test-Grundlage für einen neu-entwickelten Messsensor dar, und ein Hangrutschexperiment liefert in mehrfacher Hinsicht grundlegende Informationen zur Entwicklung numerischer Modelle. Die gemeinsame Erkenntnis aller vorgestellten WSL-Forschungsarbeiten ist, dass Experimente eine unabdingbare Brückenfunktion zwischen der reinen Beobachtung (Monitoring), der Verbesserung des Prozessverständnisses und schlussendlich der numerischen Modellierung ausüben.
\end{abstract}

\section{Einleitung}

In der Umweltforschung nehmen Experimente eine vielschichtige Rolle ein, wenn es darum geht, neues Prozesswissen zu schaffen. Was genau sich im Bodeninneren oder in der Schneedecke abspielt, wird oft erst durch gezielte Experimente verständlich. Bei Experimenten nehmen wir Forschende bewusst Einfluss, legen die Randbedingungen fest und definieren den Anfangszustand, bevor wir die Natur sich selbst überlassen. Ganz formell versteht man unter Experimenten ,das planmässig und kontrollierbar angeordnete Ablaufen lassen eines Ereignisses, mit dem Ziel, dadurch die herrschenden Gesetzmässigkeiten messbar, oder wenigstens sichtbar, zu machen. “ (Kelterborn, 1994). Sie können im Labor (unter künstlichen Rahmenbedingungen) oder im Feld (unter natürlichen Rahmenbedingungen) stattfinden, sie können in stark reduzierter Skala auf wenigen Metern oder in voller Grösse durchgeführt werden, und sie können einmalig sein oder zahlreiche Wiederholungen beinhalten.
Experimenten sind aber oft auch Grenzen gesetzt. Sie müssen sich auf einen räumlichen Ausschnitt beschränken und können niemals die volle Komplexität der Landschaft berücksichtigen. Ausserdem repräsentieren sie nur einzelne, und nicht alle möglichen Situationen (Szenarien), die eintreffen könnten. Trotzdem sind sie für unseren Kenntnisgewinn bzgl. Umweltprozessen unabdingbar.

Mit diesem Artikel möchten wir die Rolle und den Stellenwert von Experimenten näher beleuchten, indem wir einige aktuelle Beispiele aus den Forschungsarbeiten der Eidg. Forschungsanstalt für Wald, Schnee und Landschaft (WSL) aufzeigen. 


\subsection{Laborexperiment zur Kurvenüberhöhung von Murgängen}

Murgänge sind ein schnell fliessendes Gemisch aus Wasser und einem hohen Anteil an Feststoffen (Feinmaterial, Steine, Blöcke und teilweise Holz), welches in der Regel nur von aussen, d.h. mittels Fernerkundung beobachtet werden kann. Murgänge treten selten und überraschend auf, was ihre Beobachtbarkeit zusätzlich erschwert. Damit wir die Gefährdung durch Murgänge richtig beurteilen können, braucht es Wissen über ihre Eigenschaften und ihr Fliessverhalten.

Langjährige Feldbeobachtungen z.B. im Illgraben bei Susten, Kanton Wallis (vergl. Abschnitt 3), haben uns dazu wichtige Erkenntnisse geliefert. So wissen wir heute, dass in Murgängen komplexe Wechselwirkungen zwischen festen und flüssigen Phasen auftreten, wobei die feste Phase, meist bestehend aus unsortiertem Material (fein bis grobe Sedimente, Schwemmholz), die gleiche Fliessgeschwindigkeit wie die flüssige Phase (Wasser und suspendierte Sedimentpartikel) aufweist. Anderseits verbleiben zahlreiche offene Fragen zur Interaktion zwischen den verschiedenen Komponenten eines Murgangs und den daraus resultierenden Fliesseigenschaften. Zum Beispiel bilden eine breite Kornverteilung der festen Phase sowie der suspendierte Feststoffanteil an der flüssigen Phase häufig die Ursache für unterschiedliche Fliesseigenschaften.

Gerade auch was das Verhalten von Murgängen in Kurven betrifft, gibt es nur wenige Erkenntnisse. Die Anwendung eines klassischen Ansatzes aus der Hydraulik, der sogenannten „,forced vortex equation“, erlaubt uns, aufgrund von Spuren von Murgangereignissen Rückschlüsse auf die Fliessgeschwindigkeit $v$ zu ziehen (Hungr et al., 1984; Prochaska et al., 2008).

$v=\sqrt{ } \frac{\operatorname{Rg} \Delta h}{B k}$

In Gleichung (1) bezeichnet $R$ den zentralen Kurvenradius, $g$ die Erdbeschleunigung und $B$ die Breite des Gerinnes an der Stelle der beobachteten bzw. gemessenen Kurvenüberhöhung $\Delta h$. Der Korrekturfaktor $k$ entspricht einem dimensionslosen empirischen Wert zur Charakterisierung des Fliessverhaltens eines Murganges. Es sei anzumerken, dass im Falle $k=1$ die Fliesseigenschaft des Murganges dem von Wasser gleichzusetzen ist. Wie jedoch die Fliessgeschwindigkeit genau mit der beobachteten Kurvenüberhöhung $\Delta h$ und vor allem der Materialzusammensetzung des Murgangs im $\mathrm{Zu}-$ sammenhang steht, kann aus Feldbeobachtungen nicht abgeleitet werden.

Aus diesem Grund wurde im Grossraumlabor der WSL ein Experiment entwickelt, welches das Verhalten von Murgängen in Kurven systematisch untersuchen sollte (Scheidl et al., 2014). Das physikalische Modell (Abb. 1) ist einer Rutsche ähnlich, bestehend aus einem Startbehälter und einer

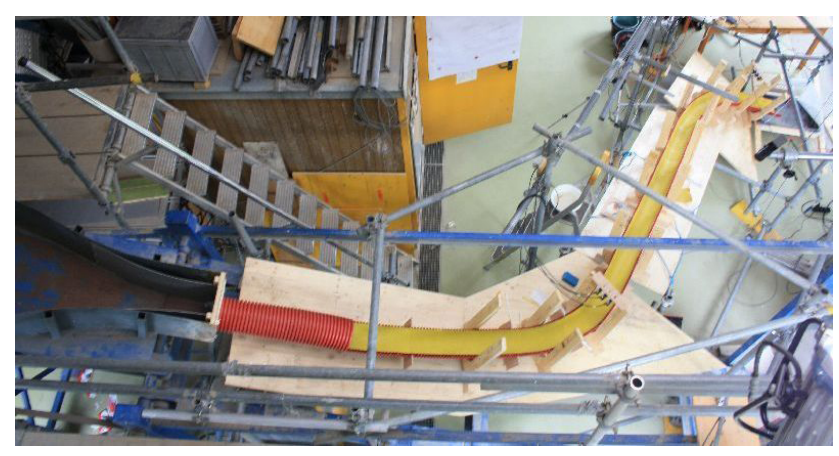

Abb. 1. Design des Experiments zur Untersuchung der Kurvenüberhöhung von Murgängen im Grossraumlabor der WSL. Am linken Rand ist der Startbehälter mit anschliessender Beschleunigungsstrecke zu erkennen. Daran anschliessend die Messstrecke mit zwei Richtungsänderungen unterschiedlicher Kurvenradien.

Messstrecke, in der das Material abfliesst. Der Startbehälter am oberen Ende der Rutsche enthält das Murgangmaterial, welches infolge der Schwerkraft nach Öffnen des Behälters durch die Messstrecke fliesst. Die Messstrecke besteht aus einem halbkreisförmigen Rohr mit einem Durchmesser von $0.17 \mathrm{~m}$ und hat eine Länge von ca. $8 \mathrm{~m}$. Zur Gewährleistung einer konstanten Rauigkeit wurde die gesamte Rinne des Modells mit Sandpapier der Körnung 40 ausgelegt. Die Messstrecke weist zwei Kurven mit Radien von $1.5 \mathrm{~m}$ (obere Kurve) und $1.0 \mathrm{~m}$ (untere Kurve) auf. Der Öffnungswinkel für beide Kurvenabschnitte beträgt $60^{\circ}$. Um der Komplexität eines Murganges Rechnung zu tragen, wurden die Materialmischungen (Feststoffe und Wasser) variiert. Es konnten 69 Versuche mit einer Neigung von $20^{\circ}$ sowie 16 Versuche mit einer Neigung von $15^{\circ}$ durchgeführt werden. Für jeden dieser Versuche wurden die maximale Kurvenüberhöhung (untere bzw. obere Kurve) sowie die mittlere Frontgeschwindigkeit im Kurvenabschnitt gemessen.

Aufgrund unserer Modellversuche zeigte sich, dass sowohl der Kurvenradius als auch die Gerinneneigung einen signifikanten Einfluss auf den Korrekturfaktor $k$ (vgl. Gleichung 1) haben. Auch konnte eine grössere Kurvenüberhöhung für Experimente mit einem hohen granularen Anteil in der Materialmischung beobachtet werden. Die Experimente zeigten ausserdem eine Abhängigkeit des Korrekturfaktors mit dem Strömungszustand des experimentellen Murganges, angenähert über die Froude-Zahl. Analysen ergaben, dass der Korrekturfaktor Werte zwischen $1<k<5$ für den überkritischen Fliesszustand (Froude $>1$ ) annimmt. In diesem Bereich scheint die „forced vortex equation“ für Murgänge anwendbar. Im Bereich des unterkritischen Fliesszustandes (Froude < 1) variiert der Korrekturfaktor und es scheint, als ob die materialbezogenen Eigenschaften eines Murganges die flüssigkeitsbedingten Eigenschaften überlagern. 


\subsection{Experiment zum Geschiebetransport in Wildbächen}

„Case studies“, d.h. detaillierte Beobachtungen an einem bestimmten, gut instrumentierten Ort, nehmen in der Umweltforschung eine wichtige Rolle ein. Die WSL betreibt beispielsweise Prototyp-Anlagen zur Beobachtung von Lawinen (Sovilla et al., 2008), von Murgängen (Hürlimann et al., 2003, vgl. Kapitel 3) und von Geschiebetransport in Wildbächen (Rickenmann et al., 2012), welche wichtig sind für die Gewinnung von wertvollen Erkenntnissen zu diesen Prozessen. Hier gewinnen die Forschenden wertvolle Erkenntnisse zu diesen Prozessen. Aber in jedem Fall bleibt die Einschränkung, dass es sich hier bezüglich Klima, Topographie und Material (Boden, Schnee, Vegetation) um eine spezifische Situation handelt. Daher steht immer die Frage im Raum, wie diese spezifischen Erkenntnisse generalisiert werden können. Experimente sind hierfür ein zentrales Hilfsmittel.

Wir möchten dies anhand von Geschiebemessungen in Fliessgewässern aufzeigen. Seit ca. 30 Jahren misst die WSL im Erlenbach, einem Wildbach in der Zentralschweiz, mit Geophonsensoren die akustischen Signale (Rickenmann und Fritschi, 2010), welche durch den Aufprall von Geschiebe auf eine Bodenplatte generiert werden, und berechnet daraus die zeitliche Dynamik des Geschiebetransports. Der Bezug zwischen den gemessenen Signalen und dem effektiven Geschiebe wurde ursprünglich anhand von Bilanzmessungen hergestellt, welche jährlich (oder nach besonderen Hochwasserereignissen) unterhalb der Messstelle in einem Geschiebesammler durchgeführt wurden. Natürlich lag die Vermutung nahe, dass dieser Bezug zwischen Signal und Geschiebe ortsspezifisch sei, d.h. abhängig von den Zuströmungsverhältnissen und von der lokalen Montage. Damit aber diese Messtechnik unabhängig von Standort generelle Aussagen über den Geschiebetransport (Menge, Kornzusammensetzung) machen kann, werden systematische Versuche mit verschiedenen Geschiebe-Eigenschaften und Durchflussgeschwindigkeiten durchgeführt. Beispielsweise haben erste Experimente stattgefunden, um den Einfluss der Konform und der Korngrösse auf das Geophonsignal zu untersuchen (Turowski und Rickenmann, 2009).

Damit die Ergebnisse ins Feld übertragen werden können, ist der in der Versuchsrinne verwendete Geophonsensor identisch eingebaut wie die Feldsensoren im Erlenbach. Für die Kalibrierung der Anlage wird die Anzahl der durch die Geophone registrierten Impulse $I$ herangezogen. In einer Studie konnte gezeigt werden, dass diese vom Geophonsignal abgeleitete Grösse am besten mit dem gesamten transportierten Geschiebematerial $M$ korreliert $\left(r^{2}=0.77-0.99\right.$; Rickenmann et al., 2014).

Das Durchführen von Experimenten in der Versuchsrinne ermöglicht es uns, den Einfluss verschiedener Parameter wie Kornform, Korngrösse, Sohlenrauigkeit und Fliessgeschwindigkeit auf das produzierte Geophonsignal zu untersuchen. Die bisher erhaltenen Versuchsresultate bestätigen die Annahme, dass diese physikalischen und hydraulischen

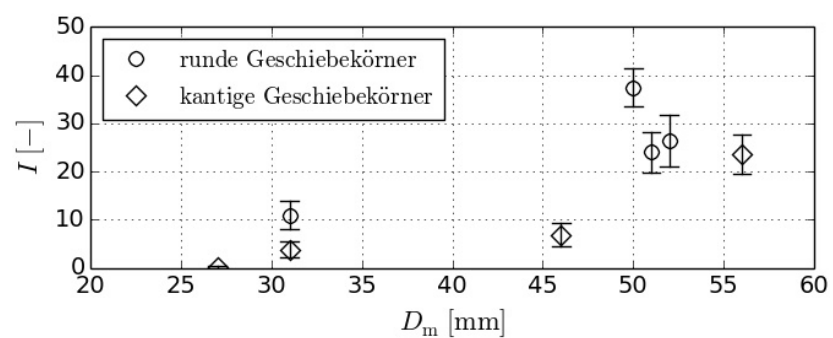

Abb. 2. Impulse $I$ in Abhängigkeit der Korngrösse für zwei verschiedene Geschiebekornformen. Diese Laborversuche wurden bei einer glatten Sohle und einer mittleren Fliessgeschwindigkeit von $1 \mathrm{~ms}^{-1}$ durchgeführt (adaptiert aus Turowski und Rickenmann, 2009).

Parameter die Anzahl der aufgezeichneten Impulse beträchtlich beeinflussen. Abbildung 2 illustriert beispielsweise den Einfluss der Kornform auf das Geophonsignal. Zu diesem Zweck wurden gezielte Experimente mit runden Geschiebekörnern und solche mit kantigen Körnern durchgeführt. Das Diagramm zeigt, dass die runden Körner über verschiedene Korngrössen ( $x$-Achse) deutlich mehr Impulse verursachen. Das kantigere Geschiebematerial hat die Tendenz, über die glatte Sohle der Versuchsrinne zu gleiten. Diese Transportform führt zu einer niedrigen Anzahl von Aufprallen auf der Geophonplatte und somit zu einer tendenziell kleinen Anzahl Impulse. Im Gegensatz dazu werden die runden Geschiebepartikel mehrheitlich rollend durch die Rinne transportiert, prallen dabei regelmässig auf die Sohle auf und übertragen mehr Energie auf die Geophonplatte. Dies resultiert in einer höheren Anzahl Impulse.

Wie aus obigem Beispiel ersichtlich, sind Laborrinnen besonders gut dazu geeignet, den Einfluss individueller Parametergrössen auf den Geschiebetransport unabhängig zu untersuchen. Solche Versuche werden in der Experimentalforschung schon seit geraumer Zeit durchgeführt (z.B. MeyerPeter und Muller, 1948). Dennoch nimmt die WSL bei der indirekten Geschiebetransportmessung mittels Geophonsensoren in Laborrinnen eine Pionierrolle ein.

\subsection{Neues Verfahren zur Untersuchung von Sturzprozessen (Steinschlag)}

Die technischen Möglichkeiten zur Beobachtung/Messung von Naturgefahrenprozessen haben in den letzten Jahren rasante Fortschritte gemacht. Innovative Sensoren zur Fernerkundung oder in situ Untersuchung von Massenbewegungen sind entwickelt worden, welche ganz neue Einblicke in die Prozessabläufe und -materialien ermöglichen. Bei der Entwicklung dieser neuen Messsysteme und Sensoren spielen Experimente eine grundlegende Rolle.

Dies soll am Beispiel von neuesten Forschungsarbeiten zu Steinschlagtrajektorien aufgezeigt werden. Die WSL untersucht schon seit einigen Jahren die Reichweite von Stein- 

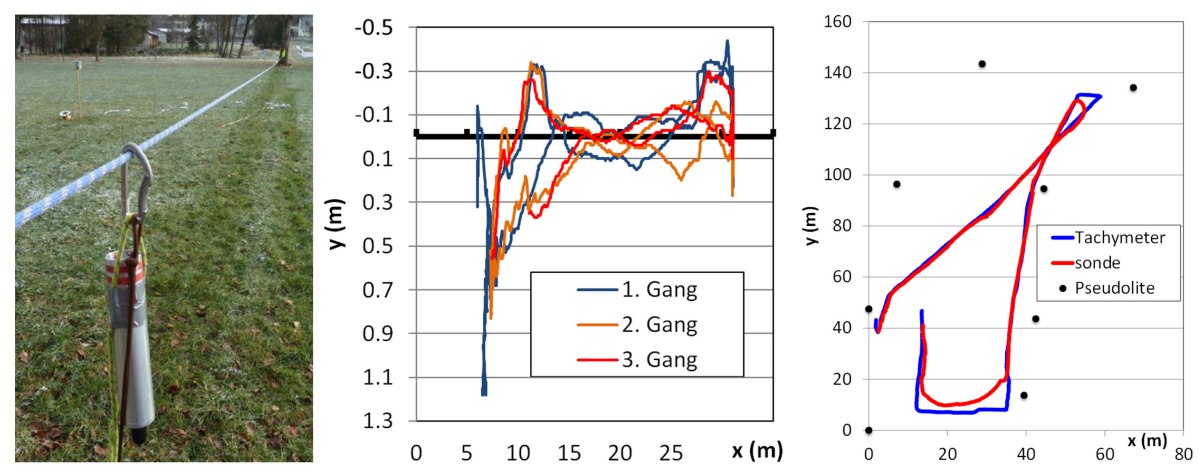

Abb. 3. Perfekt geradlinige Führung der Messsonde (links) mit der Abweichung (m) zur Referenzmessung (Mitte) und freie Bewegung zusammen mit der Referenzmessung (rechts).

schlägen und verwendet dazu u.a. Spuren im Feld und Berechnungen der Flugbahn. Effektiv gemessene Trajektorien von Steinblöcken hat es bisher nur anhand von Videoauswertungen gegeben (Dewez et al., 2010). Videoaufzeichnungen benötigen jedoch immer eine freie Sicht auf das sich bewegende Objekt und ziehen sehr langwierige Auswertungen nach sich.

Nun wurde ein neuer Sensor entwickelt, der die Flugbahn von Steinen direkt im Feld und in Echtzeit messbar machen soll. Basis dieses Sensors ist ein sogenanntes Local Positioning System (LPS). Dieses funktioniert ähnlich wie und doch umgekehrt zu GPS: Eine Sonde, welche in einen (natürlichen) Stein eingesetzt wird, sendet WiFi-Signale. Mindestens vier um das Untersuchungsgebiet angeordnete Empfängereinheiten (sog. Pseudoliten) erfassen diese Signale. Anhand der Laufzeitunterschiede kann die Position des Senders berechnet werden. Zusätzlich in die Sonde integrierte dreidimensionale Beschleunigungs- und Drehratensensoren erfassen weitere Elemente einer Steinschlagtrajektorie.

Es stellt sich die Frage, ob ein solches System überhaupt sinnvoll in einer Steinschlaguntersuchung eingesetzt werden kann. Für die zusätzlichen Sensoren existieren allgemein gültige Kalibrier- bzw. Validierungsverfahren, sodass die gemessenen Drehraten, Beschleunigungsspitzen und ganz wichtig - eine korrekte Zeitachse kontrolliert werden können. Das Hauptaugenmerk liegt darum auf dem LPS. Die Evaluierung wurde in zwei Studien vorgenommen (Klette, 2013). Eine Installation des gesamten LPS auf einem Feld ermöglicht das mehrfache Abschreiten einer idealen Gerade oder die freie Bewegung innerhalb des durch die Pseudoliten aufgespannten Polygons (Abb. 3). Diese Bewegungen werden parallel durch einen Tachymeter automatisch verfolgt und sind damit auf den Zentimeter genau referenziert.

Im zweiten Schritt wurden Steinschlagversuche im Feld durchgeführt. Dabei rollte ein $70 \mathrm{~kg}$ schwerer Stein mehrfach einen ca. $110 \times 30 \mathrm{~m}$ grossen Versuchshang hinunter (Abb. 4). Die Flugbahnen wurden gleichzeitig mit Hochgeschwindigkeitsvideo aufgezeichnet und die beobachteten Einschlagpunkte zusätzlich mit dem Tachymeter aufgenommen.
Es zeigte sich, dass das verwendete LPS durchaus für Feldversuche geeignet ist. Die Flugbahnen liessen sich mit $10 \mathrm{~Hz}$ aufzeichnen. Ein Versuchsfeld mit einer Grösse von $250 \times 50 \mathrm{~m}$ konnte problemlos von den LPS-Pseudoliten abgedeckt werden. Die Abweichungen auf der geraden Messtrecke liegen im Dezimeterbereich. Lediglich, wenn der Versuchskörper sich den Polygonrändern des Pseudolitenfeldes annähert, treten grössere Abweichungen auf. Bei der freien Bewegung fällt auf, dass das LPS enge Richtungsänderungen ausrundet. Für normale Steinschlagtrajektorien werden diese jedoch eher weniger erwartet und fallen darum nicht ins Gewicht. Die Auswertung der Feldversuche zeigte eine Abweichung der gemessenen Feld-Trajektorien zur beobachteten von bis zu $1.5 \mathrm{~m}$. Geht man nun also davon aus, dass sich mit dem System und bei einem vertretbaren Aufwand eine statistisch auswertbare Anzahl von Flugbahnen ermitteln lässt, so wird die erreichbare Genauigkeit als ausreichend angesehen. Das vorliegende LPS liefert die Position des Steines nur in 2-D, welche jedoch bei vorhandem Höhenmodell im GIS in das Gelände eingepasst werden kann. Sprunghöhen des Steins lassen sich damit jedoch nicht ermitteln. Zudem erfordert die Einschränkung auf 2-D eine einigermassen gleichmässige Neigung des Versuchshanges.

Zusammenfassend lässt sich sagen, dass mithilfe von klar definierten Experimenten eine neue Messmethode in der Steinschlagforschung Eingang gefunden hat. Mit dem Einsatz des LPS muss somit bei gross angelegten Feldstudien zukünftig nicht mehr jede einzelne Trajektorie vollständig per Video ausgewertet werden.

\subsection{Hangrutschexperiment und daraus abgeleitete numerische Modelle}

Experimente sind selbstverständlich eine essentielle Grundlage bei der Entwicklung von numerischen Modellen, und zwar in mehrfacher Hinsicht. Zum Einen können sie bei der Konzeptionierung eines numerischen Modells wichtige Hinweise und Ideen liefern. Zweitens werden Experimente zur reinen Bestimmung von Materialeigenschaften verwendet, 

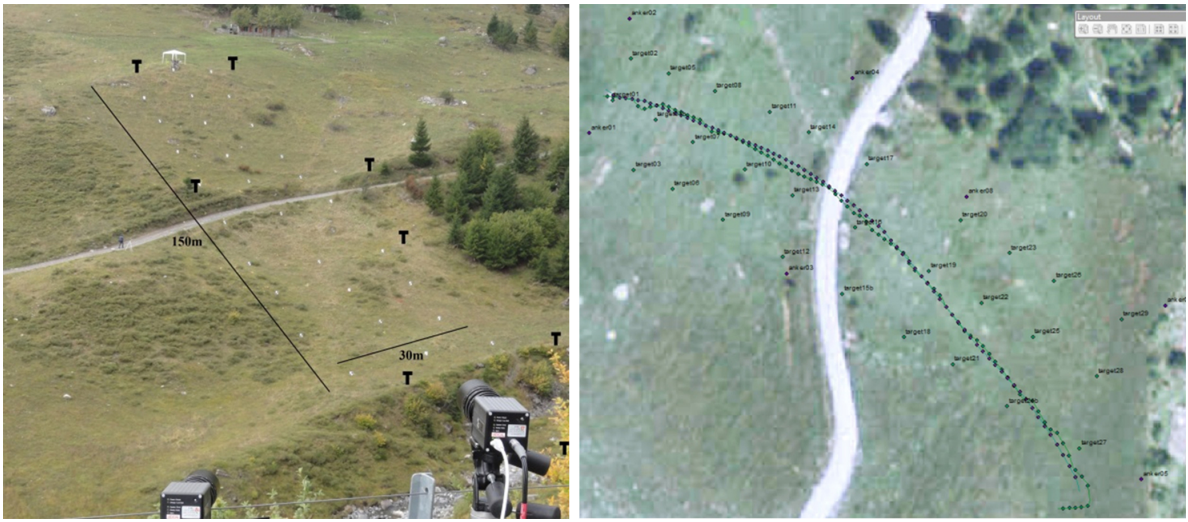

Abb. 4. Versuchshang (links, ca. $110 \times 30 \mathrm{~m}$, ca. $30^{\circ}$ ) und Überlagerung von beobachteter und gemessener Flugbahn im GIS (rechts).

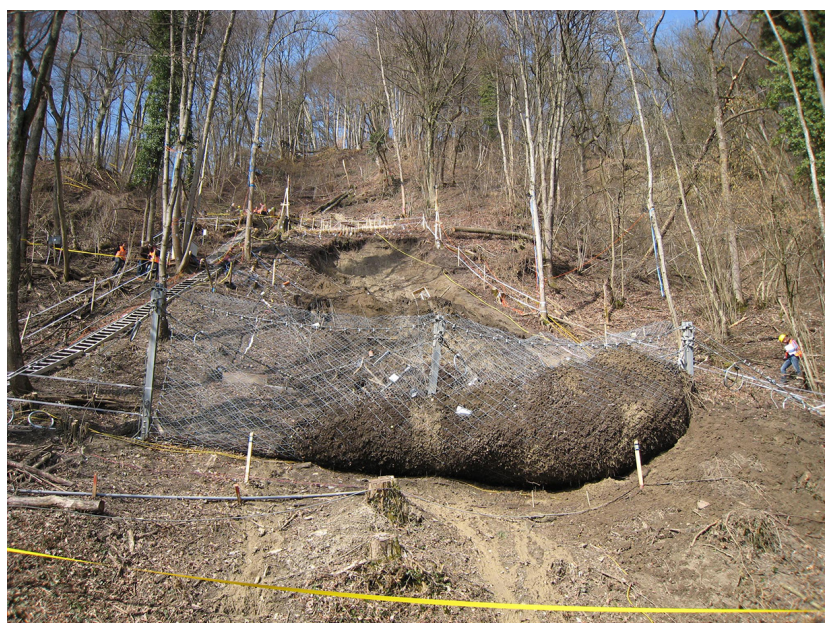

Abb. 5. Hangrutschexperiment in Rüdlingen (SH) im März 2009. (Foto: Ch. Rickli)

welche als Input ins Modell einfliessen. Und schliesslich dienen sie der Überprüfung (Validierung) der Modelle.

Ein aktuelles Beispiel zum Thema flachgründige Hangrutschungen soll dies illustrieren. Im Rahmen eines Projektes des ETH Kompetenzzentrums für Umwelt und Nachhaltigkeit (CCES) untersuchen verschiedene Gruppen der ETH Zürich, EPF Lausanne und WSL, was genau passiert, wenn sich flachgründige Hangrutschungen auslösen. Lehmann und Or (2012) haben dabei ein ganz neuartiges numerisches Modell entwickelt, das auf der Theorie der Selbstorganisierten Kritikalität (SOC) und einem Faserbündelmodell basiert, welches den Bruch von Wurzeln und Bodenelementen anhand von statistisch verteilten Schwellenwerten berechnet. Als konzeptionelle Idee für das SOC-Modell diente ein bekanntes Experiment von Bak et al. (1987), welches das lawinenartige Kollabieren eines Sandhügels aufzeigte, sobald eine bestimmte Kritikalität überschritten wurde. Das zweidimensionale Modell von Lehmann und Or verbindet benachbarte Bodenzellen über mechanische Verbin- dungen, die über eine gewisse Festigkeit verfügen. Wiederum dienten Laborexperimente (Wurzel-Zugexperimente und Boden-Scherexperimente) zur Festlegung dieser mechanischen Eigenschaften (Schwarz et al., 2011). Schliesslich wurde das Modell anhand eines Hangrutschexperiments (Springman et al., 2009) getestet. Dabei wurde an einem steilen Hang am Fusse des Rheins (Rüdlingen, $\mathrm{SH}$ ) ein $150 \mathrm{~m}^{3}$ grosser Rutsch alleine durch künstliche Beregnung ausgelöst (Abb. 5). Ein umfangreiches, vorinstalliertes Messsystem lieferte umfassende Informationen zur Aufsättigung und zur Destabilisierung des Hanges.

\section{Experimente zwischen Monitoring und Modell am Beispiel der WSL-Murgangforschung}

Quantitative Messdaten zu Murgängen in Bewegung existieren weltweit nur wenige. Dies hängt damit zusammen, dass die Prozessart einerseits eher selten und unerwartet auftritt, und andererseits die direkte Messung von Prozesseigenschaften und die damit verbundene Instrumentierung von Murganggerinnen nicht einfach ist. Die enorme Energie von Murgängen macht die Positionierung von Messgeräten anspruchsvoll.

Numerische Modelle bilden den natürlichen Prozess in einer mathematisch vereinfachten aber möglichst korrekten Form ab. Solche Modelle helfen bei der Evaluation von Sicherheitsmassnahmen (Bemessung von Schutzbauten) und beim Erstellen von Gefahrenkarten. Je nach Komplexität des Modells sind generalisierte oder sehr detaillierte Eingangsgrössen zu bestimmen. Die Ereignischarakteristik (Mischungszusammensetzung, Anzahl Schübe, etc.) eines „Designmurgangs“ für die Berechnungen wird anhand der verfügbaren Informationen im jeweiligen Einzugsgebiet bestimmt. Im Normalfall werden verschiedene Szenarien für unterschiedliche Zustände oder Wahrscheinlichkeiten gerechnet.

Die Murgangforschung wurde in der Schweiz nach den Schaden bringenden Hochwasserereignissen und Murgän- 
gen im Sommer 1987 intensiviert. Viele Fragen rund um Auslöse-, Fliess-, sowie Ablagerungsmechanismen konnten damals im Hinblick auf zu treffende Massnahmen nicht ausreichend beantwortet werden. Mit grossem Aufwand wurden seither zahlreiche Ereignisse dokumentiert und analysiert (u.a. Rickenmann und Zimmermann, 1993). Unter der aufkommenden Diskussion zu möglichen Folgen einer künftigen Klimaänderung wurden die Ereignisdokumentationen bereits damals bezüglich Vermutungen und Einschätzungen zum zukünftigen Auftreten von Murgangereignissen in Wert gesetzt (Haeberli et al., 1991). Zudem begann in dieser Zeit parallel dazu eine rege Aktivität im Bereich von ersten tauglichen Murgangmodellansätzen (u.a. Hungr, 1995). Grundlegende Überlegungen zur Modellparametrisierung für alpine Verhältnisse sind bereits im Rahmen des NFP31 Programms „Klimaänderungen und Naturkatastrophen“ formuliert worden (Zimmermann et al., 1997) und haben immer noch weitgehend Gültigkeit.

Die Erfassung von Daten und die Interpretation stattgefundener Ereignisse lässt zahlreiche Fragen zum zeitlichen Ablauf und dem Fliessverhalten von Murgängen unbeantwortet. Anhand von Abflussspuren und der Analyse des abgelagerten Materials sind nur Vermutungen zum wahrscheinlichen Ablauf möglich. Eine Rekonstruktion des Ereignisses kann nur im qualitativen Sinn und nicht mit präzisen Kennzahlen erfolgen.

Mit einer automatischen Beobachtungsstation können im Ereignisfall gezielt Messinstrumente gestartet werden, die den Prozessablauf für verschiedene Parameter vollständig aufzeichnen. Durch einen reduzierten dauerhaften Betrieb der Aufzeichnungsgeräte werden zudem die Zustände vor und nach dem Ereignis kontinuierlich dokumentiert.

Doch welche Daten aus Feldexperimenten oder von Monitoringstationen sind nun für die numerische Modellierung eines Murganges von Interesse? Moderne numerische Modelle verlangen Angaben zur erwarteten Abflussmenge, der Zusammensetzung sowie teils einer Vielzahl von weiteren Eingabeparametern (z.B. Anzahl Schübe, mögliche Veränderung der Gerinnegeometrie), welche für ein Einzugsgebiet anhand von mehr oder weniger detailliert dokumentierten Ereignissen festgelegt werden können. Um solche Modelle zu kalibrieren und generell gültige Wertebereiche für verschiedene Murgangmischungen oder Einzugsgebietstypen festzulegen, sind Datensätze von Murgangbeobachtungsstationen eine wertvolle Datengrundlage.

Die WSL hat ab den 1990er Jahren damit begonnen, an typischen Murganggerinnen automatische Murgangbeobachtungsstationen aufzubauen. Mit dem gezielten Monitoring einzelner Wildbäche mit Murgangaktivität sollen mehr Daten über die Fliesseigenschaften von Murgängen im alpinen Raum gesammelt werden, wie dies bereits einige Jahre zuvor in Asien (u.a. Itakura et al., 2005; Zhang, 1993) und den USA (Keefer et al., 1987) gemacht und zeitgleich auch in Europa gestartet wurde (Arattano, 1999). Wo erste Stationen mehr der visuellen Dokumentation der Ereignisse dienten, wur- den nach und nach Messinstrumente ergänzt, welche zentrale Kenngrössen wie etwa das Propagieren der Front ermitteln konnten. Hierzu werden Erschütterungsmessungen mit Geophonen entlang dem Gerinne durchgeführt und die Impulse pro Zeiteinheit ausgewertet (Abancò et al., 2012). Liegen zusätzlich visuelle Daten von Videokameras vor, sind neben der Front, wo typischerweise die grössten Komponenten mitgeführt werden, auch nachfolgende Einzelblöcke oder weitere Schübe auswertbar. Weitere klassische Messgeräte sind Radar oder Laser zur Bestimmung der Abflusshöhen und Rückrechnung der Abflussmengen (Hürlimann et al., 2003). Für die Bestimmung der Auslösebedingungen werden Niederschlagsmessgeräte eingesetzt. Die meisten Messgeräte verwenden berührungslose Messverfahren. Auftretende Kräfte sind in der Regel zu gross, um direkt in der Mischung zu messen oder Messinstrumente mit einem Murgang mitfliessen zu lassen.

Mit zunehmender Instrumentierung und verbessertem Prozessverständnis u.a. dank der (automatischen) Beobachtungen wurden weitere Ideen und Messmethoden entwickelt. So wurde z.B. am Illgraben bei Susten (VS) Ende 2003 eine sog. Murgangwaage (Abb. 6) ergänzt, welche mittels DMSWägezellen (DMS: Dehnmessstreifen) auf einer Wägeplatte von $8 \mathrm{~m}^{2}$ das Gewicht eines abfliessenden Murganges zeitlich hochauflösend ( $2 \mathrm{kHz}$ ) erfasst. Durch den Einbezug von Messungen der Abflusstiefe und Fliessgeschwindigkeit kann auf die Dichte des Gemisches aus Wasser und Gestein entlang der Murgangwelle zurückgeschlossen werden (McArdell et al., 2007). Bisher hatte man die Dichte eines Murgangs entweder durch die optische Auswertung von Bildmaterial abgeschätzt oder durch Entnahme und Analyse von abgelagertem Murgangmaterial nachträglich bestimmt. Bei der Entnahme von Material während einem Ereignis können neben den technischen Schwierigkeiten nur punktuelle Einblicke bei beschränkter Korngrösse erfolgen und bei der Entnahme nach einem Ereignis ist in der Regel bereits eine Änderung des Wassergehalts eingetreten. Mit der Murgangwaage wurde nicht nur die direkte Ermittlung der Dichte, sondern auch die fortlaufende Messung der Veränderung des Geschiebe- und Wasseranteils während der Dauer des gesamten Murgangs möglich, was für das Prozessverständnis und für die Entwicklung von numerischen Simulationsmodellen sehr wichtige Informationen liefert.

Als weiteres Beispiel für innovative Weiterentwicklung und gezieltes Messdatenerfassen kann z.B. der sog. Laservorhang im Dorfbach Randa (VS) genannt werden (Graf et al., 2013; Abb. 7). Damit wird mittels neuer Lasertechnologie ein Querprofil mit hoher Frequenz (bis $100 \mathrm{~Hz}$ ) gescannt. Die Abflusskurven einzelner Murschübe werden im Abstand von ca. einem Meter in Fliessrichtung sehr genau aufgezeichnet, und es besteht die Möglichkeit, mittels einer Korrelationsanalyse von sichtbaren Partikeln an der Oberfläche zwischen den beiden nahe beieinanderliegenden Querprofilen eine sowohl zeitlich als auch örtlich fein aufgelöste Oberflächengeschwindigkeit zu bestimmen. Daneben kann in Kennt- 


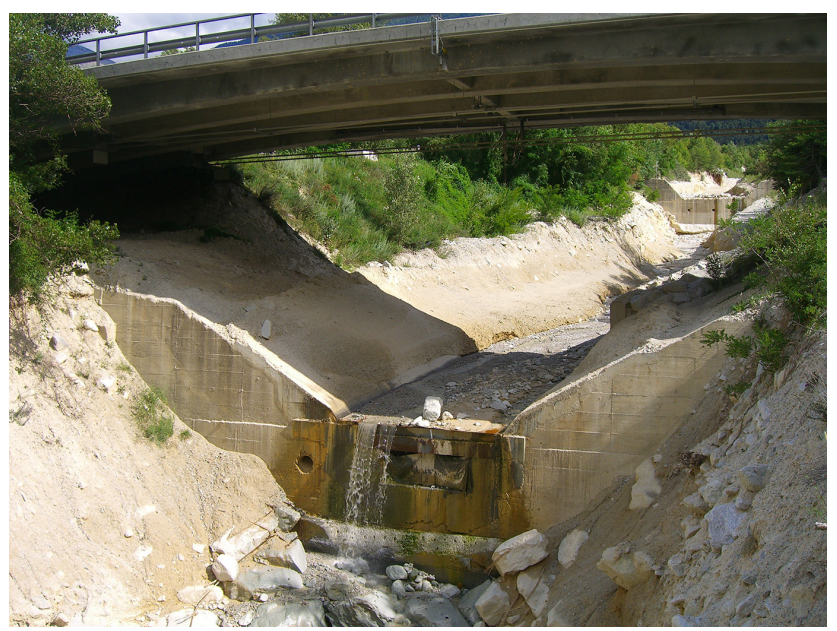

Abb. 6. Murgangwaage im Illgraben (VS) im Juni 2007 nach dem Durchgang eines Murganges. (Foto: F. Dufour)

nis der Geometrie vor und nach einem Ereignis die laterale Verteilung der Fliesstiefe abgeschätzt werden. Über die Integration des momentanen Abflusses erfolgt die Bestimmung des Volumens eines Murschubes. Die regelmässige Messung der Profile erlaubt zudem die Quantifizierung von Erosion und Deposition an der Messstelle. Mit Hilfe dieser neuartigen Datensätze können unter anderem numerische Modelle wie RAMMS (RApid Mass Movement System; Christen et al., 2012) noch präziser kalibriert und damit die Auslaufmodellierung verbessert werden.

Ein drittes Beispiel stellt die Erosionsmessanlage dar, die in den Jahren 2008 und 2009 im Illgraben in Betrieb war (Berger et al., 2010). Aus den Untersuchungen wissen wir genauer Bescheid über den Zeitpunkt und das Ausmass der Erosion am Gerinnebett. Diese findet typischerweise in den ersten Sekunden beim Durchgang der Front statt. Neben den sog. Erosionssensoren, einer Säule aus erodierbaren Rohrelementen, die über eine Widerstandsleitung miteinander verbunden sind, ermittelt eine Druckmessung am unteren Ende der Säule den aktuellen Porendruck im Gerinnebett (Abb. 8). Daraus abgeleitet ergeben sich die vertikale Veränderung der aktuellen Sohlentiefe und die Druckzustände im Untergrund zum jeweiligen Zeitpunkt. Einige wenige Ereignisse konnten mit der Anlage erfolgreich ausgewertet werden und Vermutungen über die Erosionsleistung und den zeitlichen Verlauf mit konkreten Messdaten belegen. Für die Weiterentwicklung von Modellen sind derartige Erkenntnisse von eminenter Wichtigkeit.

Die oben genannten Messeinrichtungen sind alle aus neuen Fragestellungen erwachsen, welche sich bei der Analyse von Messdaten und Beobachtungen ergeben haben. Neue Erkenntnisse im Prozessverständnis sowie das Bedürfnis nach detaillierteren Kennwerten für die Bestimmung der Eingangsgrössen von numerischen Modellen werden diese Entwicklung weiterhin steuern.

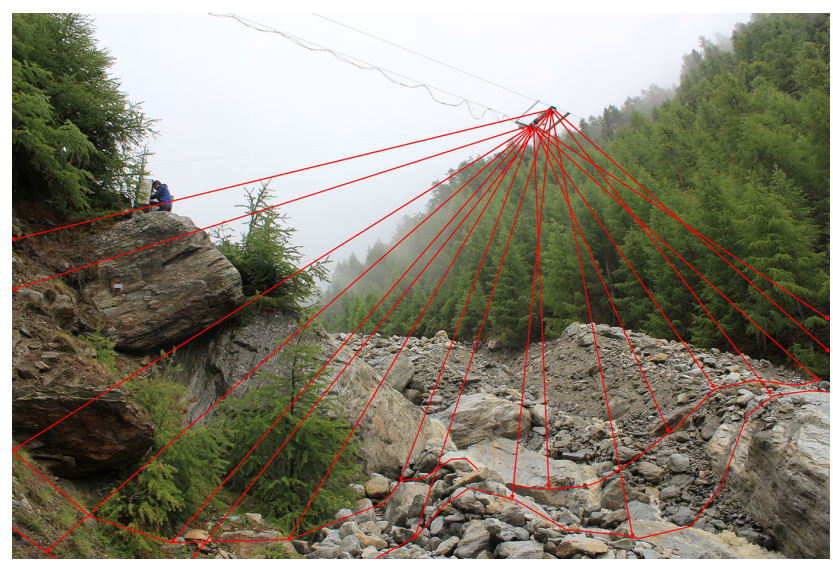

Abb. 7. Profillaserinstallation am Dorfbach Randa (VS) im Sommer 2011. (Foto: Ch. Graf)
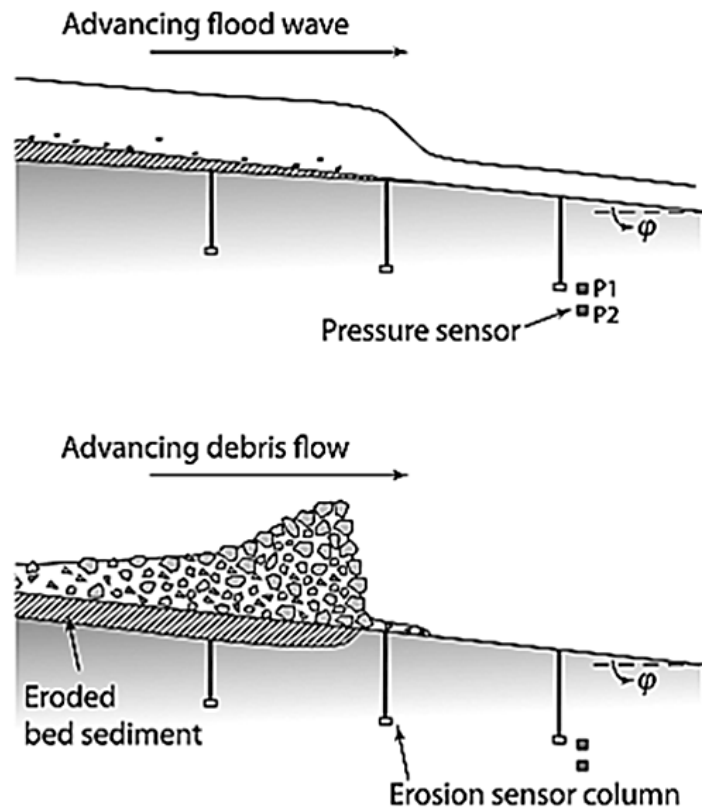

Abb. 8. Messprinzip Erosionssensoren im Illgraben (VS) nach Berger et al. (2010).

Die Positionierung und der Aufbau einer automatischen Monitoringstation zur Gewinnung von Ereigniskenndaten wird nur an ausgewählten Standorten ins Auge gefasst. Die Installationen sind meist kostenintensiv. Ein Standort muss garantieren, dass in gewisser Regelmässigkeit ein Ereignis stattfinden wird. Zudem ist es wünschenswert, wenn er zumindest eine gewisse regionale Repräsentativität aufweisen. Meist orientiert sich die Standortwahl an kürzlich stattgefundenen Ereignissen oder ist begründet durch klare Hinweise auf eine mögliche Intensivierung von Ereignissen, etwa durch sich stark verändernde Ausgangsbedingungen im Einzugsgebiet. Es bleibt jedoch immer offen, mit welcher Frequenz und Magnitude Ereignisdaten erfasst werden können. 
Der Aufwand für den Betrieb automatischer Murgangbeobachtungsstationen und die Auswertung der anfallenden Daten darf nicht unterschätzt werden. Die Tätigkeiten erfordern sowohl finanzielle Mittel für die technischen Geräte und deren Unterhalt sowie für die Arbeitsleistung. Standorte sollten über mehrere Jahre bis Jahrzehnte betrieben werden, um eine genügend grosse Datengrundlage zu erfassen. Die sinnvolle Dauer wird massgeblich durch die Frequenz der Ereignisse mitbestimmt.

Immer mehr werden Erfahrungen aus langjähriger instrumentierter Murgangbeobachtung in Anlagen zur Warnung und Alarmierung von besonders gefährdeten Zonen (z.B. Siedlungen, Strassen- und Bahnabschnitten) eingesetzt. Daraus ergeben sich wiederum Möglichkeiten, neue Messtechnologien an den Forschungsstationen zu testen und die Verfahren für die Auswertung der Daten zu optimieren, damit diese zu einem späteren Zeitpunkt ebenfalls in operationellen Systemen zum Einsatz kommen können.

Zusammenfassend können wir behaupten, dass heute dank den zahlreichen Messungen von Murgängen in Bewegung die Bandbreite von realistischen Kennwerten genauer bekannt sind. Wir wissen, wann Erosion stattfinden kann und wie weit Murgänge auslaufen können. Sämtliche Daten liefern uns Grundlagen für die mathematische Nachbildung der Prozessart und die Beurteilung ihrer Resultate. Schliesslich wissen wir heute dank diesen Messungen, dass jedes Ereignis seine Eigenheit aufweist, was Prognosen hinsichtlich dem zeitlichen und örtlichen Auftreten sowie der Ausprägung nach wie vor ausserordentlich schwierig macht.

\section{Fazit}

Die diskutierten Beispiele der WSL machen deutlich, dass Experimente in der Naturgefahrenforschung nie einen Selbstzweck verfolgen, sondern eine wichtige Funktion zum quantitativen Prozessverständnis erfüllen. Sie zeigen auch, dass Experimente ideale Spielfelder für technische Messinnovation darstellen. Und schliesslich offenbaren sie die Notwendigkeit von Experimenten für die Entwicklung von numerischen Modellen.

Trotzdem stellen wir fest, dass die experimentelle Erkundung von Murgängen, Hangrutschungen und Steinschlag zur Zeit (in der Schweiz) eher in reduziertem Masse stattfindet. Das hängt sicher u.a. mit den hohen technischen Anforderungen solcher Versuche und vielen offenen Fragen bei der Übertragbarkeit der Ergebnisse auf die komplexen FeldSituationen zusammen. Dennoch sind wir der Meinung, dass die zahlreichen guten Erfahrungen der letzten Jahre eine erneute Intensivierung der Experimente in diesem Forschungsfeld durchaus rechtfertigen.

Danksagung. Die in diesem Artikel beschriebenen Experimente konnten nur dank einem grossen Einsatz zahlreicher Mitarbeitender der Eidg. Forschungsanstalt WSL realisiert werden. Ein besonderer
Dank geht an Bruno Fritschi, der die meisten elektronischen Messsysteme der erwähnten Experimente konzipiert und gebaut hat. Wir bedanken uns ausserdem bei der Solcon Systemtechnik GmbH, Lübeck, für die Entwicklung des in Kapitel 2.3 beschriebenen Sensors. Finanziell wurden die Experimente vom Schweizerischen Nationalfonds (Grant Nr. 200021_137681/1), ETH Kompetenzzentrum für Umwelt und Nachhaltigkeit CCES, Österreichischem Wissenschaftsfonds FWF (J 3089-N13) sowie durch die WSL unterstützt. Schliesslich möchten wir den verschiedenen kommunalen, kantonalen und eidgenössischen Behörden danken, welche diese Arbeiten unterstützt haben.

Edited by: P. Greenwood

Reviewed by: two anonymous referees

\section{Literatur}

Abancó, C., Hürlimann, M., Fritschi, B., Graf, C., und Moya, J.: Transformation of ground vibration signal for debris-flow monitoring and detection in alarm systems, Sensors, 12, 4870-4891, 2012.

Arattano, M.: On the use of seismic detectors as monitoring and warning systems for debris flows, Nat Hazards, 20, 197-213, 1999.

Bak, P., Tang, C., und Wiesenfeld, K.: Self-Organized Criticality: An Explanation of 1/f noise, Phys. Rev. Lett., 59, 381-384, 1987.

Berger, C., McArdell, B. W., Fritschi, B., und Schlunegger, F.: A novel method for measuring the timing of bed erosion during debris flows and floods, Water Resour. Res., 46, W02502, doi:10.1029/2009WR007993, 2010.

Christen, M., Bühler, Y., Bartelt, P., Leine, R., Glover, J., Schweizer, A., Graf, C., McArdell, B. W., Gerber, W., Deubelbeiss, Y., Feistl, T., und Volkwein, A.: Integral hazard management using a unified software environment: numerical simulation tool "RAMMS" for gravitational natural hazards, edited by: Koboltschnig, G., Hübl, J., and Braun, J., 12th Congress INTERPRAEVENT, 23-26 April 2012 Grenoble - France, Proceedings, Vol. 1, Klagenfurt, International Research Society INTERPRAEVENT, 77-86, 2012.

Dewez, Th., Nachbaur, A., Mathon, C., Sedan, O., Berger, F., und Des Garets, E.: OFAI: 3D block tracking for a real-size rockfall experiment in the weathered volcanic context of Tahiti, French Polynesia, Geophysical Research Abstracts, Vol. 12, EGU20104104, 2010.

Graf, C., Deubelbeiss, Y., Bühler, Y., Meier, L., McArdell, B., Christen, M., und Bartelt, P.: Gefahrenkartierung Mattertal: Grundlagenbeschaffung und numerische Modellierung von Murgängen, in: Mattertal - ein Tal in Bewegung, edited by: Graf, C., Publikation zur Jahrestagung der Schweizerischen Geomorphologischen Gesellschaft 29 Juni-1 Juli 2011, St. Niklaus. Birmensdorf, Eidg. Forschungsanstalt WSL. 85-112, 2013.

Haeberli, W., Rickenmann, D., Rösli, U., und Zimmermann, M.: Murgänge; Ursachenanalyse der Hochwasser 1987, Ergebnisse der Untersuchungen, Bern, Mitteilung des Bundesamtes für Wasserwirtschaft, Bern, Nr. 4, Mitteilung der Landeshydrologie und -geologie, Bern, Nr. 14, 1991.

Hungr, O.: A model for the runout analysis of rapid flow slides, debris flows, and avalanches, Can. Geotech. J., 32, 610-623, 1995. 
Hungr, O., Morgan, G., und Kellerhals, R.: Quantitative analysis of debris torrent hazards for design of remedial measures, Can. Geotech. J., 21, 663-677, 1984.

Hürlimann, M., Rickenmann, D., und Graf, C.: Field and monitoring data of debris-flow events in the Swiss Alps, Can. Geotech. J., 40, 161-175, 2003.

Itakura, Y., Inaba, H., and Sawada, T.: A debris-flow monitoring devices and methods bibliography, Nat. Hazards Earth Syst. Sci., 5, 971-977, doi:10.5194/nhess-5-971-2005, 2005.

Keefer, D. K., Wilson, R. C., Mark, R. K., Brabb, E. E., Brown, W. M., Ellen, S. D., Harp, E. L., Wieczorek, G. F., Alger, C. S., und Zatkin, R. S.: Real-time landslide warning during heavy rainfall, Science, 238, 921-925, 1987.

Kelterborn, P.: Was ist ein wissenschaftliches Experiment?, AEASAnzeiger, 1, 7-9, 1994.

Klette, J.: Evaluierung einer Messtechnik zur Erfassung von Steinschlagflugbahnen. Diplomarbeit. Technische Universität bergakademie Freiberg, 2013.

Lehmann, P. und Or, D.: Hydromechanical triggering of landslides: From progressive local failures to mass release, Water Resour. Res., 48, W03535, doi:10.1029/2011WR010947 2012.

McArdell, B., Bartelt, P., und Kowalski, J.: Field observations of basal forces and fluid pore pressure in a debris flow, Geophys. Res. Lett., 34, L07406, doi:10.1029/2006GL029183, 2007.

Meyer-Peter, E. und Muller, R.: Formulas for bed-load transport, Proceedings of 2nd Meeting Int. Assoc. Hydraul. Struct. Res., 39-64, 1948.

Prochaska, A., Santi, P., Higgins, J., und Cannon, S.: A study of methods to estimate debris flow velocity, Landslides, 5, 431-444, 2008.

Rickenmann, D. und Fritschi, B.: Bedload transport measurements using piezoelectric impact sensors and geophones, Bedloadsurrogate Monitoring Technologies, edited by: Gray, J. R., Laronne, J. B., and Marr, J. D. G., US Geological Survey Scientific Investigations Report 2010-5091, 2010.

Rickenmann, D. und Zimmermann, M.: The 1987 debris flows in Switzerland: documentation and analysis, Geomorphology, 8, 175-189, 1993.
Rickenmann, D., Turowski, J. M., Fritschi, B., Klaiber, A., und Ludwig, A.: Bedload transport measurements at the Erlenbach stream with geophones and automated basket samplers, Earth Surf. Process. Landforms, 37, 1000-1011, 2012.

Rickenmann, D., Turowski, J. M., Fritschi, B., Wyss, C. R., Laronne, J. B., Barzilai, R., Reid, I., Kreisler, A., Aigner, J., Seitz, H., und Habersack, H: Bedload transport measurements with impact plate geophones: comparison of sensor calibration in different gravel-bed streams, Earth Surf. Process. Landforms, 39, 928942, 2014.

Scheidl, C., McArdell W. B., und Rickenmann, D.: Debris-flow velocities and superelevation in a curved laboratory channel, Can. Geotech. J., doi:10.1139/cgj-2014-0081, published online, 2014.

Schwarz, M., Cohen, D., und Or, D.: Pullout tests of root analogs and natural root bundles - experiments and modeling, J. Geophys. Res., 116, F02007, doi:10.1029/2010JF001753, 2011.

Sovilla, B., Schaer, M., Kern, M., und Bartelt, P.: Impact pressures and flow regimes in dense snow avalanches observed at the Vallée de la Sionne test site, J. Geophys. Res., 113, F01010, doi:10.1029/2006JF000688, 2008.

Springman, S. M., Kienzler, P., Casini, F., und Askarinejad, A.: Landslide triggering experiment in a steep forested slope in Switzerland, Proceedings of the 17th International Conference on Soil Mechanics and Geotechnical Engineering, edited by: Maza, M., Hamza, M., Shahien, M., and El-Mossallamy, Y., doi:10.3233/978-1-60750-031-5-1698, 2009.

Turowski, J. M. und Rickenmann, D.: Tools and cover effects in bedload transport observations in the Pitzbach, Austria, Earth Surface Process. Landforms, 34, 26-37, doi:10.1002/esp.1686, 2009.

Zhang, S.: A comprehensive approach to the observation and prevention of debris flows in China, Nat. Hazards, 7,1-23, 1993.

Zimmermann, M., Mani, P., Gamma, P., Gsteiger, P., Heiniger, O., und Hunziker, G.: Murganggefahr und Klimaänderung - ein GISbasierter Ansatz [debris flow risk and climatic change - a GISbased approach]. Zürich, vdf Hochschulverlag AG an der ETH Zürich, Switzerland, 1997. 